\title{
Psicoterapia de Pareja ante el descubrimiento del involucramiento del varón en actividades sexuales por Internet
}

\section{Couple Psychotherapy in front of the discovery of male involvement in sexual activities on the Internet}

\author{
Diana Rivera-Ottenberger \\ Escuela de Psicología, Pontificia Universidad Católica de Chile, Chile \\ Cristóbal Hernández Contreras \\ Escuela de Psicología, Universidad Adolfo Ibáñez, Chile \\ Rec (10 de Abril de 2017) Acept (17 de Marzo de 2020)
}

\begin{abstract}
Resumen
El desarrollo tecnológico de Internet ha facilitado la conectividad entre personas con motivaciones muy diversas. Una de ellas, es acceder a contenidos sexuales y conectarse con otros que comparten este interés. El uso de internet con fines sexuales incluye una amplia gama de comportamientos, algunos de los cuales pueden generar una crisis en una relación de pareja estable y comprometida, al ser significados como una infidelidad. Las características propias del medio y de los usuarios de internet con fines sexuales, desafía a los terapeutas a adquirir nuevas herramientas psicoterapéuticas para ayudar a las parejas en conflicto. El artículo tiene por objetivo a) dar a conocer los principales conceptos vinculados al uso de internet con fines sexuales (b) describir las características del cyber affair y comportamientos significados como infidelidad (c) establecer lineamientos psicoterapéuticos para el tratamiento de este tipo de casos.

Palabras clave: Internet, sexo, pornografía, cyber affair, psicoterapia de pareja
\end{abstract}

\begin{abstract}
The technological development of the Internet has facilitated the connectivity between people with very diverse motivations. One is accessing sexual content and connecting with others who share this interest. The use of the Internet for sexual purposes includes a wide range of behaviors, some of which can generate a crisis in a stable and committed couple relationship, being signified as an infidelity. The characteristics of the media and Internet users for sexual purposes, challenges therapists to acquire new psychotherapeutic tools to help couples in conflict. The article aims to a) to disclose the main concepts related to the use of the Internet for sexual purposes (b) to describe the characteristics of the cyber affair and behaviors signified as infidelity (c) to establish psychotherapeutic guidelines for the treatment of this type of cases.
\end{abstract}

Key words: Internet, sex, pornography, cyber affair, couple psychotherapy

Correspondencia: Diana Rivera-Ottenberger dvrivera@uc.cl. Dirección Postal: Avda. Vicuña Mackenna 4860, Macul. Santiago de Chile

Agradecimientos: Este estudio recibió apoyo del Fondo de Innovación para la Competitividad (FIC) del Ministerio de Economía, Fomento y Turismo, a través de la Iniciativa Científica Milenio, Proyecto IS130005. 


\section{Introducción}

El acceso masivo a Internet ha posibilitado la ampliación de la conectividad entre las personas y un mayor acceso a la información. La sexualidad es uno de los ámbitos del comportamiento humano que ha sido muy impactado por este medio. De hecho, el sexo es uno de los tópicos que concita mayor búsqueda entre los usuarios (Nua Internet Surveys, 2003).

La internet posee tres características que facilita su uso con fines sexuales, denominadas "Motor Triple A" (Triple-A Engine): fácil acceso (accesibility), anonimato (anonymity) y bajo costo (affordability) (Cooper, 1998). A estas tres características Hertlein y Piercy (2012) agregan cuatro más, la aceptación de internet como moderador de la comunicación (acceptable), la facilitación de acercamientos a interacciones de la vida real (approximation), la ambigüedad para establecer o definir comportamientos problemáticos (ambiguity) y finalmente, permitir grados variables de acomodación respecto del self real y los deseados (accomodation).

La investigación en este tópico ha seguido básicamente dos lineamientos (Cooper, Scherer, Boies, \& Gordon, 1999a). La primera, enfatiza los aspectos patológicos, riesgosos y negativos del medio, como por ejemplo, facilitar la realización de comportamientos sexuales desviados o criminales como generar y difundir material pornográfico infantil. La segunda, destaca los aspectos positivos del uso del medio (e.g. información sobre sexualidad saludable) y como modalidad de exploración sexual acorde a los tiempos actuales y de conectividad entre las personas (e.g., búsqueda de pareja, grupos de apoyo).

Si bien no todos los comportamientos sexuales en internet son problemáticos, cada vez es más frecuente que las parejas heterosexuales, traigan como motivo de consulta el consumo de sexo por internet por parte del varón. Lo anterior es comprensible, pues los hombres usan más pornografía que las mujeres, la utilizan a menor edad y la emplean con mayor frecuencia mientras se estimulan para obtener gratificación sexual (Fisher \& Byrne, 1978). A su vez, los varones ven pornografía más frecuentemente solos o con amigos, mientras que las mujeres la usan más habitualmente con su pareja sexual (Hald, 2006). Las mujeres por su parte, son más críticas con este material y lo consideran con más frecuencia que los varones, tonto, repulsivo o simplemente poco excitante (Træen, Spitznogle, \& Beverfjord, 2004).

La importancia del tema para terapeutas e investigadores se debe primeramente, a que la infidelidad — en línea o fuera de ella — tiene efectos negativos en la salud mental de quien fue engañado. A modo de ejemplo, en el caso de las mujeres sin antecedentes de depresión, la probabilidad de deprimirse posterior al descubrimiento de la infidelidad de su pareja, es de un 38\% (Christian-Herman, O'Leary, \& Avery-Leaf, 2001), probabilidad que aumenta a un $72 \%$, en aquellas con antecedentes de depresión (Cano \& O’Leary, 2000). Por otra parte, la infidelidad vía internet crecientemente es motivo de ruptura de las relaciones de pareja (Schneider, 2003; Whitty \& Quigley, 2008), siendo necesario buscar modalidades psicoterapéuticas efectivas para las parejas que solicitan ayuda (Hertlein et al., 2012). Esta realidad probablemente va ir en aumento en la medida que Chile es uno de los países de Sudamérica donde internet ha tenido una cobertura creciente (Internet World Stats, 2016). A su vez, si bien en las dos últimas décadas se han realizado estudios sobre las características de los usuarios y los efectos en las parejas y familias del uso de internet con fines sexuales (Schneider, 2000, 2002), la investigación y el tratamiento de las infidelidades vía internet es un campo que recién se inicia (Ayres \& Haddock, 2009; Hertlein et al., 2012; Young, Griffin-Shelly, Cooper, O’Mara, \& Baucham, 2000).

El artículo tiene por objetivo (a) describir los principales conceptos vinculados al uso de internet con fines sexuales (b) describir los efectos del uso de pornografía y del cyber affair en la relación de pareja (c) plantear lineamientos psicoterapéuticos básicos para el tratamiento de estas parejas.

\section{Aspectos conceptuales}

\section{Sexualidad por Internet}

La actividad sexual por internet (Online Sexual Activity, OSA) es un concepto amplio que incluye cualquier actividad por internet (incluyendo texto, audio o archivos gráficos) que involucre la sexualidad, ya sea con fines recreacionales, de exploración, educacionales, búsqueda de pareja u otros (Cooper, McLaoughlin, \& Campbell, 2000; Leiblum \& Döring, 2002). 


\section{Cybersex}

Este concepto, es una subcategoría de OSA, y puede ser definido como el empleo de internet como medio para involucrarse en actividades sexuales gratificantes, como son, el mirar imágenes, participar en chat sexuales, intercambiar imágenes sexuales explícitas o e-mails, compartir fantasías a través de internet mientras las personas obtienen gratificación sexual ("cybering"), entre otros (Cooper, 2004).

\section{Sexting}

Refiere al comportamiento específico de enviar, recibir o reenviar mensajes, imágenes o fotos sexualmente explícitas por medios electrónicos, especialmente por medio de teléfonos celulares; es realizado preferentemente por jóvenes (Kholos \& Childers, 2011; Klettke, Hallford, \& Mellor, 2014). También puede incluir el envío de videos cortos.

\section{Pornografía}

La definición de un material como pornográfico está sujeta a grandes variaciones históricas y culturales (Sabo, 2009). Con fines operacionales Hald (2006) la definió como "Cualquier material destinado a crear o promover pensamientos y sentimientos sexuales en quien los recepciona y que, al mismo tiempo (1) contenga la descripción y/o la exposición explícita de los genitales y (2) actos sexuales claros y explícitos como relaciones vaginales, relaciones anales, sexo oral, masturbación, esclavitud, sadomasoquismo, violación, sexo con orina, sexo con animales, etc." (p. 579). Según este autor (Hald), los materiales que muestran a hombres y mujeres posando desnudos, tal como se ve en revistas como Playboy o Playgirl, y que no incluyen actos sexuales claros y explícitos, no debe considerarse material pornográfico. Otros autores destacan más bien la finalidad sexualmente excitatoria del material, "Material que se encuentra destinado o se espera que genere excitación sexual en quién lo recibe" (Allen, Emmers, Gebhardt, \& Giery, 1995, p. 13). Sin embargo, estas definiciones se complejizan si se considera distintos tipos de pornografía (e.g., entre personas de igual o distinto sexo, con animales, niños, o de contenido violento). Manning (2006) destaca la insuficiencia de las definiciones anteriores, porque no incorporan elementos comunes en este tipo de material, que incluye degradación, subordinación, violencia hacia las mujeres, entre otros, sosteniendo que en la clínica es de mayor utilidad conocer los parámetros de evaluación de los consultantes.

\section{Cyber affair}

Refiere al establecimiento de una relación sexual y/o emocional vía online y que se inicia y mantiene predominantemente a través del contacto virtual, vía e-mail o participando en comunidades virtuales, como chat rooms, juegos interactivos, o newsgroups (Millner, 2008; Young et al., 2000). Incluye comportamientos con grados variables de intimidad sexual y emocional (e.g., compartir fantasías sexuales y satisfacción sexual mutua). Esta relación puede progresar paulatinamente hacia un encuentro cara a cara, especialmente cuando el comportamiento supone involucramiento emocional, más que sexual (Henline, Lamke, \& Howard, 2007).

\section{Comportamientos sexuales y tipos de usuarios de internet}

La forma en que las personas utilizan internet y los comportamientos sexuales asociados a ello son variados y van desde un rango normal a uno problemático (Cooper et al., 1999a). A modo de ejemplo, los usuarios pueden mirar imágenes o vídeos con material sexual, intercambiar e-mails o imágenes de contenido explícitamente sexual, buscar información y leer material sexual, involucrarse en chateos sexuales, compartir fantasías sexuales entre desconocidos, buscar a personas que quieran involucrarse sexualmente en forma personal, realizar comportamientos parafílicos, entre otros. Para el $92 \%$ de los usuarios que emplean internet con fines sexuales, los comportamientos que realizan no les son problemáticos (Cooper et al, 1999a).

Los usuarios de internet que acceden a contenidos sexuales pueden ser clasificados en tres categorías (Cooper, Putnam, Planchon, \& Boies, 1999b): (1) usuarios recreacionales; (2) usuarios de riesgo; y (c) usuarios sexual- 
mente compulsivos. Los usuarios recreacionales constituyen el $88 \%$ de los usuarios de sexo vía internet (Cooper et al, 1999a). Acceden a estos contenidos por diversión, curiosidad y por lo novedoso del contenido. Su fin es fundamentalmente recreativo y no les genera problemas en otras áreas de sus vidas, ni desarrollan comportamientos adictivos a estos sitios de internet. En segundo lugar, los usuarios de riesgo, corresponde a personas que, si bien tenían una vulnerabilidad latente, mantenían el control sobre su sexualidad. Sin embargo, al tener acceso a este material comienzan a tener problemas en el control de sus comportamientos y a experimentar los efectos negativos de ello, como por ejemplo, utilizar tiempo destinado al trabajo para estos fines, deteriorar su relación de pareja o destinar mucho dinero a esta actividad, afectando los ingresos familiares o personales. Dentro de esta categoría, se distinguen dos sub tipos (Cooper et al., 1999b), el subtipo 1 o tipo depresivo, que frecuentemente presenta depresión, distimia o ansiedad, y el subtipo 2 o tipo stress reactivo, que usa internet sobre todo en los períodos de alto estrés para aliviar la tensión y las emociones desagradables asociadas a ello. Cuando el período de alto estrés declina, tienden a limitar el acceso a internet y vuelven a utilizar las estrategias adaptativas que anteriormente empleaban. Tienen la capacidad de establecer relaciones cercanas y muchas veces reconocen estos comportamientos como problemáticos y solicitan ayuda (Cooper et al., 1999b). El tercer tipo, corresponde a los usuarios sexualmente compulsivos, quienes ya antes del consumo presentaban una propensión hacia una sexualidad compulsiva (e.g., múltiples parejas ocasionales, interés sistemático por la pornografía, sexo telefónico, prostitución, parafilias). El sexo compulsivo tiene las características de los desórdenes de control de impulsos, es decir, la persona experimenta la intensa necesidad de realizar un determinado comportamiento sin poder controlarlo, a pesar de las consecuencias adversas en el plano relacional, laboral, económico o de otro tipo (Cooper, Delmonico \& Burg, 2000b). Estos aspectos son comunes a los desórdenes de control de impulsos y de las adicciones, pero en estas últimas, se evita las consecuencias adversas de no realizar el comportamiento y se siente placer en su concreción. Esto último ha llevado a discutir si estos comportamientos deben o no ser incorporados en la clasificación de los comportamientos adictivos en manuales ampliamente difundidos, como por ejemplo el Diagnostic and Statistical Manual of Mental Disorders (DSM) (Gold \& Heffner, 1998; Goodman, 1992, 2001). Algunos indicadores de presencia de sexo compulsivo vía internet son: (1) ocupar más de 11 horas semanales en actividades sexuales en internet; (2) interferencia de esta actividad en otras áreas de la vida (laboral, financiera) o en las relaciones interpersonales (distanciamiento); (3) falseamiento de la identidad que se vincula con aquello que excita (e.g., cambio de sexo, edad) y (4) sentimientos de culpa y vergüenza con los comportamientos. Estas personas representan aproximadamente el $8 \%$ de los usuarios de internet con fines sexuales y difieren del resto, en que porcentualmente mantienen menos relaciones matrimoniales o de pareja estable, evidencian una tendencia al aislamiento y la soledad, participando más de chateos y medios interactivos que los otros grupos (ver Cooper et al., 1999a). Autores como Adams y Robinson (2001) destacan más que el aspecto adictivo o compulsivo del desorden, el aspecto vincular. Refieren que la gran mayoría de estas personas presentan serias dificultades en establecer vínculos de intimidad. Estas dificultades pueden hacerse evidentes ya en la adolescencia, donde puntuaciones elevadas en adicción a internet, se asocia con mayores niveles de ansiedad social, menores habilidades sociales y falta de asertividad (Torrente, Piqueras, Orgilés, \& Espada, 2014).

Los usuarios sexualmente compulsivos difieren de los usuarios recreacionales, en que utilizan internet como medio principal para expresar sus comportamientos sexuales, siendo su motivación la obtención de placer y el alivio de la excitación sexual. Sin embargo, los tres tipos de usuarios de internet presentan el potencial común, de transitar hacia el establecimiento de una relación amorosa cara a cara, con la persona conocida a través de internet (Cooper et al., 1999b).

Los comportamientos que las personas evalúan como una infidelidad en relación a internet son muy diversos, e incluyen por ejemplo, el cibersex, el intercambio de imágenes sexuales personales, la búsqueda de pareja, el flirteo y la pornografía on line entre otros (Henline et al., 2007; Hertlein \& Webster, 2008; Whitty, 2003). A continuación, se considerará solo dos de estos comportamientos, el uso de pornografía y el cyber affair, pues son aquellos que motivan más frecuentemente la consulta psicológica. 


\section{El descubrimiento}

\section{El uso de pornografía}

Las personas que usan internet con fines sexuales —independientemente de su motivación y finalidad - lo mantienen en su gran mayoría en secreto. Un $70 \%$ de los usuarios no comparte con otros el tiempo que dedica a esta actividad, correspondiendo el $72 \%$ a varones y el 62\% a mujeres (Cooper et al., 1999a). Por esta razón, para la mayoría de las mujeres, el descubrimiento les genera en un principio sorpresa e incredulidad, y posteriormente van variando el grado de aceptación o rechazo a estos comportamientos, dependiendo de sus marcos valóricos, el tipo e intensidad de los comportamientos que el varón ha desarrollado y el material al cual ha accedido. Efectivamente, hay estudios que evidencian que el uso de pornografía por parte de los varones tiene efectos diferenciales en las mujeres. El malestar es significativamente menor y en ocasiones no se experimenta, en relaciones de menor compromiso (e.g., recién saliendo con la pareja) y cuando el uso de pornografía es poco frecuente y por espacios breves de tiempo (Bridges, Bergner, \& Hesson-Mcinnis, 2003). El siguiente caso ilustra una modalidad de afrontamiento menos frecuente.

Caso 1: Claudia de 32 años chilena, casada desde hace 9 años, madre de dos hijos, descubre que su esposo, nacido en Europa, accede a contenidos pornográficos (heterosexuales y lésbicos) obteniendo gratificación sexual con ello. En sesión, la pareja discutió extensamente si esto era impropio, una patología, si correspondía a un problema de pareja, de familia, o sería considerado como parte de la vida privada del esposo. Ellos decidieron que él podía seguir con estas prácticas dado que, eran poco frecuentes y ambos estaban muy seguros de la estabilidad de la relación y del amor que mutuamente se profesaban.

En otras ocasiones, las mujeres aceptan este comportamiento por razones muy diversas, como aducir que lo consideran preferible a que el varón tenga un encuentro cara a cara con otra mujer, o piensan que todos los hombres usan pornografía o que, es un alivio para ellas que su pareja satisfaga de esta forma sus frecuentes deseos sexuales (Bridges et al., 2003).

Por el contrario, Bergner y Bridges (2002) señalan que, en el caso de consumo intenso de pornografía, las mujeres que descubren este hecho, mayoritariamente experimentan un gran impacto, confusión y modifican su percepción de la relación y su pareja: se sienten engañadas y traicionadas por sus parejas; se cuestionan su grado de valía y atractivo personal; experimentan intensos sentimientos de rechazo, ajenidad y desconocimiento de la pareja; y finalmente, cuestionan la integridad, valía y salud mental de la pareja

En estas ocasiones, es frecuente que las mujeres interroguen al terapeuta respecto de si estos comportamientos son normales o no, pues parecen no tener parámetros, guiones o "scripts" para evaluarlos (Escoffier, 2007). Se suma a lo anterior la discordancia en el significado e importancia que le otorgan al hecho los miembros de la pareja, pues las mujeres ven las actividades sexuales online más seriamente que los varones, lo significan más frecuentemente como una infidelidad (Parker \& Wampler, 2003) y no les agrada que sus parejas consuman pornografía (Maddox, Rhoades, \& Markman, 2011; Morgan, 2011).

Sin embargo, contrario a los antecedentes anteriores, en las parejas donde los varones comparten honestamente con su pareja que consumen pornografía, las mujeres reportan mayor nivel de satisfacción con la relación y menor malestar con la pornografía, en comparación con las mujeres cuyas parejas les habían ocultado el este hecho (Resch \& Alderson, 2014). A su vez, no existían diferencias en la satisfacción con la relación de pareja entre el grupo de mujeres que conocía el hecho y que habitualmente y siempre compartían pornografía con su pareja, comparados con el grupo que raramente o nunca lo hacía. De acuerdo a lo anterior, Resch et al., 2014 sostienen que el ocultamiento y la falta de honestidad sería uno de los factores que sí afecta la satisfacción con la relación en las mujeres, más que el uso mismo de la pornografía por parte del varón.

\section{El cyber affaire}

$\mathrm{Al}$ igual que en el caso de la pornografía, el cyber affaire o romance cibernético, lleva a discutir a la pareja —además de lo propio o impropio del comportamiento - si el consumo de sexo por internet constituye o no una infidelidad. Habitualmente los varones sostienen que el sexo cibernético no lo es, argumentando que no hubo un encuentro corporal, ni existió la intencionalidad de establecer contacto físico con esa persona, es decir "no es real". Este es un argumento comúnmente empleado, pues el encuentro físico cara a cara es lo que suele 
considerarse como infidelidad propiamente tal (Avila, 2007). Sin embargo, aun cuando no haya existido un encuentro cara a cara, la infidelidad online genera efectos similares a una infidelidad offline en la persona ofendida, como son, intenso dolor, sentimientos de traición y deslealtad, pérdida de la confianza y cuestionamiento de la continuidad de la relación (Whitty, 2005).

Un comportamiento muy común que suele considerarse una infidelidad es el flirteo online. Se caracteriza porque las personas comparten pensamientos y sentimientos íntimos, sin involucrar conversaciones directas sobre sexo, pero sí de auto exposición y coquetería en los diálogos. Esto podría catalogarse como cyber affair de acuerdo a la definición en el apartado anterior (Millner, 2008; Young et al., 2000). En estos diálogos, se produce un jugueteo, referido como "jugar al amor", donde las personas comparten aspectos íntimos con un otro, dejando abierta la expectativa de que algo amoroso puede acontecer, pero con muy pocas posibilidades de concreción (Whitty, 2003). En los textos se pueden emplear emoticones, letras mayúsculas, puntos suspensivos, u otros, en reemplazo del lenguaje no verbal, característico de la coquetería de los encuentros cara a cara. El siguiente caso, ejemplifica este hecho.

Caso 2: Juana de 58 años, solicita a su esposo Pedro de 60 años, que le preste el computador para revisar su e-mail. Descubre en su Facebook — cuya sesión ha quedado abierta — que él mantiene conversaciones intermitentes de gran intimidad emocional y de coqueteo desde hace más de un año con una ex compañera de colegio, a quien no ve desde la adolescencia. Ha compartido sentimientos y pensamientos que su esposa desconocía, tanto de sí mismo, como de la relación entre ellos. Usa términos como "que duermas bien entre tus envidiables sábanas", "con nadie he compartidos mis dolores como tú", o bien ante la pregunta de su amiga "¿me extrañas?", él responde "¡iMUUCHO!!”; o finaliza un mensaje con "Un beso"; o una carita coqueta @; o con el emoticón :-))) que significa "muchas sonrisas".

Whitty (2003) sostiene que el flirteo es una forma de juego adulto, donde existe un grado de fantasía y se encuentra por lo general separado de la "vida real". Sin embargo, esto es vivido por la pareja o esposa del varón, como una traición al espacio de intimidad y confidencia, privilegio de una relación exclusiva y de compromiso. Queda instalada en la mujer la duda de qué hubiese acontecido si no descubre el hecho, es decir, se abre la interrogante sobre el curso que podrían haber tomado esta relación y las verdaderas intenciones del esposo, que desde su perspectiva sería la concreción de una relación emocional y sexual con esta tercera persona.

Ante la interrogante de la mujer sobre las motivaciones del comportamiento del varón, es común que este refiera que no sabe por qué lo hizo, aumentando la desconfianza de la pareja, a quien le parece inconcebible una respuesta de este tipo en un hombre adulto, con quien tiene una relación de alto compromiso. Esta respuesta en parte puede ser cierta, pues las personas que experimentan en el medio, no tienen los patrones interpretativos de cómo se dan las relaciones en un contexto virtual y porque el medio permite una cierta ambigüedad para establecer o definir comportamientos problemáticos (Hertlein et al., 2012). A su vez, este tipo de comportamientos se ve reforzado porque quienes lo realizan, pues experimentan mucha diversión y excitación emocional, independientemente de si permanecen anónimos o no (Whitty, 2003). En la experiencia de los autores, estos sentimientos y experiencias son difíciles de aceptar, pues al ser expuestos los hechos ante la pareja, pueden parecer comportamientos burdos, infantiles y hasta ridículos, comparados con el riesgo al cual se expuso la relación y los efectos adversos en el bienestar de la pareja.

En otros casos, agrava la situación que el varón en estas conversaciones cambie aspectos de su identidad, como por ejemplo, su estado civil (de casado a soltero o separado), o la descripción de hobbies que le hacen más interesante (e.g., cocinero, deportista, fotógrafo) lo cual alude a la acomodación de la identidad que internet permite (Hertlein et al., 2012). Estas son formas de presentar una imagen más positiva y atractiva a un otro, pues el cuerpo vía internet toma otras expresiones o formas de representación que en los encuentros presenciales (Whitty, 2003, 2004, 2007). En estos casos es altamente posible que la relación curse desde una relación vía online, a una llamada telefónica y luego a un encuentro cara a cara (Whitty \& Gavin, 2001).

El descubrimiento de esta realidad puede deberse a un evento más bien fortuito, como en el caso anterior (Caso 2), o bien porque las mujeres notan cambios en el comportamiento de su pareja, como son, el pasar más tiempo conectado al celular o computador, utilizar estos aparatos cuando el resto de la familia duerme, solicitar la exploración de nuevos comportamientos sexuales y/o disminución del deseo sexual (Young et al., 2000). Las mujeres en una primera instancia, interpretan estos comportamientos como producto de la carga de trabajo de sus parejas o esposos, cosa que ellos habitualmente emplean como justificación, pero posteriormente comienzan a sospechar la existencia de una tercera persona, lo cual las motiva a indagar en el uso del computador y telefonía 
que el esposo hace. Una vez que confirman el uso de cybersex, la mayoría de ellas experimenta intensos sentimientos de desconocimiento del otro, desconcierto, rechazo y decepción. Esta incapacidad de comprender qué llevó a la pareja a estos comportamientos, lleva a las mujeres a promover la solicitud de ayuda terapéutica.

\section{Intervención psicoterapéutica}

En la experiencia de los autores, la gran mayoría de los varones que consultan con su pareja, corresponden a consumidores de riesgo (depresivo o estrés reactivo), seguido de consumidores recreacionales y en mucha menor medida, consumidores compulsivos.

Aun cuando no existen tratamientos validados para abordar la infidelidad mediada por internet (Hertlein et al., 2006; Vossler, 2016), existen ciertas tareas y estrategias terapéuticas sistematizadas por Hertlein et al (2012), quienes entrevistaron a 15 terapeutas con experiencia en estos temas que delinearon tareas fundamentales: (a) desarrollo de límites físicos (b) desarrollo de límites psicológicos (c) manejo de los sentimientos, la responsabilidad, y la confianza (d) tomar conciencia sobre la etiología del problema (e) evaluar el contexto de la pareja y disposición al cambio (f) evaluación de circunstancias únicas y (g) promover el trabajo hacia el perdón. A continuación, se describen las tareas y estrategias terapéuticas, acompañadas de comentarios y sugerencias de los autores.

Tarea 1 Desarrollo de límites físicos: La primera tarea psicoterapéutica, apunta a la recuperación de la seguridad y la confianza del miembro de la pareja que ha sido ofendido. Esto implica, por ejemplo, delimitar los contextos o impedir el acceso a internet de la persona que se involucró en actividades sexuales por este medio. También la pareja puede acordar que su uso sea en presencia de la persona que no participaba de estas actividades, o bien que este último pueda acceder a información que le permita saber cómo ha usado la pareja internet (e.g., eliminación de claves de acceso; no borrar historial o mensajes). Sin embargo, estas medidas pueden ser insuficientes dado que una de las característica del medio, es su fácil acceso (Cooper, 1998), incrementado por la portabilidad. Dado que la actividad sexual por internet usualmente es significada como infidelidad, no sólo es necesario abordar la recuperación de la confianza — cuestión común al tratamiento de las infidelidades - sino también promover el incremento de la intimidad y comunicación en la pareja (ver Young et al., 2000).

Tarea 2 Desarrollo de límites psicológicos: Es necesarios que la pareja discuta sobre lo que cada uno considera una infidelidad o traición a la confianza, pues los comportamientos correctos o incorrectos en internet tienen una cierta ambigüedad y la virtualidad del encuentro desdibuja su realidad. Lo que genera intenso dolor en la pareja, más que la infidelidad sexual, es el hecho del secreto, de conocer dimensiones de la pareja impensables y de haber quedado fuera de esto (Hertlein et al., 2012).

De acuerdo a la experiencia de los autores, esta tarea abre un campo de conversaciones novedoso para la pareja, pues requiere de explicitar nuevas reglas sobre lo que se puede o no hacer con otros en la red, qué tipo de información se puede o no compartir, cuáles son los límites psicológicos que permite que ambos se sientan seguros y confiados en la relación y cuáles son los límites de lo privado, entre otros. Young et al (2011) sostienen - basándose en su experiencia que en casos de adicción a internet— que el tratamiento también abre la posibilidad de reflexionar sobre los valores compartidos, pues tal como se mencionó anteriormente, la actividad sexual del varón por internet, hace que la mujer se pregunte sobre quién es esa persona, cómo ve y aprecia el mundo y si han cambiado sus valores. El abordaje de esto facilita la comunicación, la recuperación de la confianza y una puesta al día sobre la forma de apreciar la relación de pareja, la familia y los otros ámbitos en que se desenvuelve la vida de una persona (Young et al., 2011).

En la experiencia de los autores, habitualmente los consumidores recreacionales de pornografía están más dispuestos a dejar de usar este material y restablecer la confianza de su pareja. En otros casos, si bien dicen que lo harán, sus esposas quedan con la duda de aquello, perpetuando la desconfianza y el conflicto en la relación.

Tarea 3 Manejo de la responsabilidad, la confianza y los sentimientos: este punto refiere a validar activamente los sentimientos de la persona que se siente traicionada con los comportamientos de la pareja. El terapeuta puede promover la expresión de sus sentimientos y validarlos frente al compañero, facilitando la escucha de este, de modo que pueda conocer los efectos de su comportamiento en el bienestar de su la pareja y tomar responsabilidad y conciencia de ello (Hertlein et al., 2012). Sólo desde este reconocimiento y validación, se puede progresar en la elaboración del evento. En ocasiones esto puede ser difícil, pues desde la perspectiva del varón, 
la infidelidad no existe, pues no hay encuentro corporal, o bien puede haber interactuado con otra persona desde el anonimato, o bien simplemente, considerarlo irrelevante. Esto puede tomar tiempo, pero es fundamental para el curso del tratamiento.

Tarea 4 Incrementar la toma de conciencia sobre la etiología del problema: esta tarea alude a conocer las motivaciones, que en este caso tuvo el varón, para involucrarse en cybersex. Hertlein et al (2012) sostienen que los terapeutas entrevistados, colocan énfasis en detectar las necesidades insatisfechas que motivan a la persona para involucrarse en actividades sexuales por internet, aun cuando reconoce que las causas de la infidelidad son multifactoriales. Desde la experiencia de los autores, cobra especial relevancia el diagnóstico sobre el tipo de usuario del cual trata el caso, usuario recreacional, de riego o sexualmente compulsivo (Cooper et al., 1999b), pues las indicaciones terapéuticas pueden variar según esto. Efectivamente, de acuerdo a Cooper y Marcus (2003), los usuarios sexualmente compulsivos, tienen una dificultad significativa para establecer relaciones de intimidad, presentando a su vez, otras comorbilidades. Sostienen que constituyen un desafío terapéutico relevante, pues generan poderosos sentimientos contratransferenciales. Tras una fachada de seguridad y éxito en varias áreas de sus vidas, presentan intensos sentimientos de vergüenza, inadecuación y de estar fuera de control. Estos autores (Cooper et al, 2003) proponen una modalidad de tratamiento con base psicoanalítica y fundamentalmente, de tratamiento individual, basada en reparar este desorden relacional, trabajando las dificultades en el establecimiento de relaciones der intimidad, en un contexto psicoterapéutico de aceptación. Un ejemplo de este tipo de consumidores se presenta a continuación.

Caso 3: Mabel y José son una pareja de profesionales jóvenes, con un hijo de 2 años y se encuentran a la espera de su segundo hijo. Mabel desde hace un año nota cambios significativos en el comportamiento de su esposo, descubriendo que accede frecuentemente a pornografía. Emplea sexo cibernético y goza sexualmente con ello, lo que ha distanciado sus relaciones sexuales. El esposo se queda hasta altas horas de la madrugada en estas actividades y en ocasiones la requiere sexualmente después de ello, cosa que a ella le molesta, sintiéndose usada y humillada. José en entrevista individual, refiere que con sus amigos han conformado un grupo donde comparten material pornográfico, acceden a nuevos sitios de internet y últimamente han comenzado a asistir regularmente a centros nocturnos donde hay desnudos, y posibilidades de acceder a la prostitución, consumo de alcohol y sustancias. Tiene algo de conciencia que está en vista a acceder a este tipo de sexo. Presenta un consumo excesivo de marihuana desde que era adolescente y abusa con frecuencia del alcohol cuando sale con sus amigos. Tanto en su familia de origen y extensa, existen antecedentes de adicciones diversas. Luego de promover la apertura de su situación con su esposa — de la cual ella en parte conocía - se realizan 8 sesiones conjuntas, donde finalmente se acuerda derivar a José a terapia individual y atención con un especialista en adicciones, dado el aumento paulatino de comportamientos sexuales de riesgo y su actuar compulsivo, muchas veces bajo el efecto de sustancias. Se les plantea que posteriormente se evaluará la continuación de la terapia de pareja.

En la experiencia de los autores, otro de los comportamientos habituales por los cuales consultan las parejas, es el flirteo. Este comportamiento se ve facilitado por la amplia red de personas a las cuales se puede acceder vía internet (e.g., Facebook), dentro de las cuales pueden estar personas con las cuales alguna vez existió alguna atracción o relación amorosa. Para indagar las motivaciones que tuvo la persona para involucrarse en sexo cibernético en el caso del flirteo, se pueden emplear los parámetros motivacionales propios de las relaciones cara a cara, que son: (a) facilitar el contacto sexual (motivación sexual), (b) para avanzar hacia la concreción de una relación de pareja (motivación relacional), (c) por diversión (motivación de diversión), (d) para explorar la potencialidad de una relación romántica (motivación de explorar), (e) para aumentar la autoestima (motivación de autoestima), y (f) para animar a otros a hacer algo por la persona (motivación instrumental) (Henningsen, 2004; Henningsen, Braz, \& Davies, 2008). En experiencia de los autores, la utilización de estas motivaciones y su profundización en la indagatoria, son útiles para el flirteo tanto con y sin anonimato.

Tarea 5 Evaluar el contexto de la pareja y disposición para el cambio: el consumo de sexo por internet, se da en un contexto biográfico y en un momento vital personal y de pareja, lo cual queda claramente expuesto en el caso anterior (Caso 3). Dentro de la evaluación de la relación y de cada uno de sus miembros, se puede indagar sobre las habilidades comunicacionales, habilidades de negociación, el grado en que disfrutan de su relación, la historia previa de infidelidades, no solo entre ellos, sino que también a través de las generaciones; se sugiere también evaluar las expectativas acerca de la relación y el grado de compromiso de cada persona con su promoción y mantenimiento. Esta información facilita el establecimiento de las metas del tratamiento, tanto individuales, como de la pareja (Hertlein et al., 2012). 
De acuerdo a la experiencia de los autores, además de la exploración del contexto de la pareja, en el caso de los consumidores de riesgo y compulsivos, es conveniente realizar una interconsulta psiquiátrica para evaluar el uso paralelo de tratamiento medicamentoso (e.g., ansiolíticos y/o antidepresivos) y explorar las formas en que los varones enfrentan situaciones estresantes y/o penosas, particularmente con su pareja, junto a su capacidad de vinculación. Este fenómeno se relaciona con una de las razones del uso de internet, la búsqueda de modificar el estado de ánimo, siendo uno de ellos, el evitar la experimentación de eventos internos privados y desagradables como son, sensaciones corporales, memorias, pensamientos y emociones perturbadoras (Caplan, 2002). La evitación de estas experiencias, puede mantener o exacerbar el uso problemático de sexo por internet, constituyéndose en una forma problemática de regulación emocional (Grant \& Potenza, 2006). El siguiente caso es un ejemplo de ello.

Caso 4. Rodrigo de 47 años, casado hace 15 años con Constanza de 39 años, cuatro hijos. Él pierde su trabajo hace diez meses y se encuentra trabajando en casa en forma independiente, muy agobiado por las responsabilidades familiares como único proveedor. De temperamento ansioso y algo obsesivo, comienza a acceder a pornografía para distraerse y aliviar sus tensiones. Accede a sexo cibernético y paulatinamente comienza a conectarse con mayor frecuencia con una persona desconocida, quien ocupa cada vez más tiempo en sus pensamientos. Su esposa descubre esta situación, porque sospecha una infidelidad. Durante el tratamiento reconoce su angustia y agobio, refiriendo que fue socializado para no pedir ayuda y que no visualiza a su esposa como fuente de apoyo emocional ni material. Se siente muy culpable, humillado y degradado por su despido. Refiere que la relación virtual que mantenía, aumentaba su autoestima y valía personal, proporcionándole alivio a través del goce sexual.

En la experiencia de los autores, es conveniente explorar cómo las personas reaccionan frente a estos sentimientos (e.g., dolor, desamparo, humillación) de acuerdo a su historia vincular actualizada en este contexto, denominado ciclo de la vulnerabilidad (ver Scheinkman \& Fishbane, 2004).

Tarea 6 Evaluación de circunstancias únicas: aquí se debe colocar atención en ciertas particularidades del caso y su asociación con el problema, como son, la presencia de adicción a internet u otra, la existencia de un desorden de personalidad, aparición de problemas de salud que afecten el desempeño sexual personal o de la pareja, evaluar las expectativas de género y su rol en la relación, y finalmente, clarificar la "real" existencia de una tercera persona.

Tarea 7 Promoción del perdón: Hertlein et al (2012) sostienen que un número relevante de los terapeutas entrevistados sugirió trabajar en la promoción del perdón. Proponen como estrategia, evaluar cuán dispuesto está el cónyuge ofendido en avanzar hacia el perdón, entendiendo que el perdón es una decisión que puede o no tomar en relación a lo acontecido. Algunas preguntas que pueden favorecer el logro de la tarea son, " ¿Qué necesita Ud. para reconstruir la confianza? " y " ¿En qué contribuirá Ud. para ser capaz de tomar la decisión de perdonar? ' (Hertlein et al., 2012, p. 266).

\section{Conclusiones}

El descubrimiento del uso de internet con fines sexuales, es un motivo de consulta cada vez más frecuente entre las parejas, lo cual es concordante con el crecimiento exponencial de las tecnologías de la información y de las comunicaciones en la sociedad contemporánea (Internet World Stats, 2016). Sin embargo, los profesionales de la salud mental que trabajan con parejas y familias, reportan tener escaso conocimiento y formación para enfrentar estos casos (Ayres et al., 2009). Esto les hace susceptibles a operar bajo sus particulares puntos de vista, a riesgo de patologizar a los usuarios y asumir sesgos de género inadecuados (Ayres et al, 2009; Hertlein et al., 2012).

El impacto en la relación de pareja de la pornografía y el cyber affair, no debe subestimarse, pues el malestar que genera en la pareja del usuario, es por lo general similar a una infidelidad cara a cara. A su vez, la infidelidad es uno de los eventos definidos como de los más humillantes en las relaciones maritales y se asocian estrechamente a la aparición y/o agravamiento de la depresión (Wisman, 2016). A la fecha hay avances en establecer diferencias entre los tipos de usuarios de internet con fines sexuales (Cooper et al., 2000b) y en establecer lineamientos para el tratamiento psicoterapéutico (Hertlein et al., 2012; Young et al, 2000). Sin embargo, esta es un área que requiere de mucha más investigación, pues el estudio del impacto de la tecnología en las rela- 
ciones interpersonales y de pareja está recién en sus pasos iniciales, existiendo escasa literatura en las revistas especializadas en terapia familiar y de pareja (Blumer, Hertlein, Smith, \& Allen, 2014).

\section{Referencias}

Adams, M. A. \& Robinson, W.R. (2001). Shame reduction, affect regulation, and sexual boundary development: Essential building blocks of sexual addiction treatment. Sexual Addiction and Compulsivity, 8(1), 23-44. doi:10.1080/10720160127559

Allen, M., Emmers, T., Gebhardt, L. \& Giery, M. A. (1995). Exposure to pornography and acceptance of rape myths. Journal of Communication, 45(1), 5-26. doi:10.1111/j.1460-2466.1995.tb00711.x.

Avila, B.L. (2007). Online infidelity in Internet chat rooms: an ethnographic exploration. Computers in Human Behavior, 23(1), 11-31. doi:10.1016/j.chb.2004.03.033

Ayres, M. M. \& Haddock, S. A. (2009). Therapists' approaches in working with heterosexual couples struggling with male partners' online sexual behavior. Sexual Addiction y Compulsivity, 16(1), 55-78. doi:10.1080/10720160802711952

Bergner, R.M. \& Bridges, A.J. (2002). The significance of heavy pornography involvement for romantic partners: research and clinical implications. Journal of Sex \& Marital Therapy, 28(3), 193-206. doi:10.1080/009262302760328235

Blumer, M.L., Hertlein, K.M., Smith, J.M. \& Allen, H. (2014). How many bytes does it take? A content analysis of cyber issues in couple and family therapy journals. Journal of Marital and Family Therapy, 40(1), 34-48. doi: 10.1111/j.1752-0606.2012.00332.x

Bridges, A. J., Bergner, R. M. \& Hesson-McInnis, M. (2003). Romantic partners' use of pornography: its significance for women. Journal of Sex \& Marital Therapy, 29(1), 1-14. doi:10.1080/713847097

Cano, A., \& O’Leary, K.D. (2000). Infidelity and separations precipitate major depressive episodes and symptoms of nonspecific depression and anxiety. Journal of Consulting and Clinical Psychology, 68(5), 774-781. doi: 10.1037//0022-006X.68.5.774

Caplan, S. E. (2002). Problematic Internet use and psychosocial well-being: Development of a theory-based cognitive-behavioral measurement instrument. Computers in Human Behavior, 18(5), 553-575. doi:10.1016/s0747-5632(02)00004-3

Christian-Herman, J., O’Leary, K.D., \& Avery-Leaf, S. (2001). The impact of negative events in marriage on depression. Journal of Social and Clinical Psychology, 20, 24-40. doi: 10.1521/jscp.20.1.24.22250

Cooper, A. (1998). Sexuality and the Internet: Surfing into the new millennium. CyberPsychology and Behavior, 1(2), $187-193$. doi:10.1089/cpb.1998.1.187

Cooper, A. (2004). Online Sexual Activity in the New Millennium. Contemporary Sexuality, 38(3), p. i-vii.

Cooper, A., Delmonico, D. \& Burg, R. (2000). Cybersex users, abusers, and compulsives: New findings and implications. En: A. Cooper (edt), Cybersex: The Dark Side of the Force (pp. 5-29). Philadelphia: Brunner: Routledge.

Cooper, A., McLoughlin, I.P. \& Campbell, K.M. (2000). Sexuality in cyberspace: Update for the $21^{\text {st }}$ century. CyberPsychology and Behavior, 3(4), 521 - 536. doi:10.1089/109493100420142

Cooper, A. \& Marcus (2003). Men who are not in control of their Sexual Behavior. En: Levine, S.B., Risen, C.B. \& Althof, S.E. (edits). Handbook of Clinical Sexuality for Mental Health, (pp. 311-332). New York: Brunner - Routledge.

Cooper, A., Putnam, D., Planchon, L. \& Boies, S. (1999b). Online sexual compulsivity: Getting tangled in the net. Sexual Addiction $y$ Compulsivity, 6(2), 79-104. doi:10.1080/10720169908400182

Cooper, A., Scherer, C. R., Boies, S. C. \& Gordon, B. L. (1999a). Sexuality on the Internet: From sexual exploration to pathological expression. Professional Psychology: Research and Practice, 30(2), 154-164. doi:10.1037/0735-7028.30.2.154

Escoffier, J. (2007). Scripting the Sex: Fantasy, narrative, and sexual scripts. En: M. Kimmel (edt). The Sexual Self (pp. 61-79). Nashville: Valderbilt University Press.

Fisher, W. A. \& Byrne, D. (1978). Sex differences in response to erotica? Love versus lust. Journal of Personality and Social Psychology, 36(2), 117-125. doi:10.1037/0022-3514.36.2.117

Gold, S. N., \& Heffner, C. L. (1998). Sexual addiction: Many conceptions, minimal data. Clinical Psychology Review, 18(3), 367-381. doi:10.1016/s0272-7358(97)00051-2

Goodman, A. (1992). Sexual addiction: Designation and treatment. Journal of Sex \& Marital Therapy, 18(4), 303-314. doi:10.1016/ s0272-7358(97)00051-2

Goodman, A. (2001). What's in a name? Terminology for designating a Syndrome of driven sexual behavior. Sexual Addiction and Compulsivity, 8(3-4), 191-213. doi:10.1080/107201601753459919

Grant, J.E. \& Potenza, M.N. (2006). Compulsive aspects of impulse control disorders. Psychiatric Clinics of North America, 29(2), 539-551. doi:10.1016/j.psc.2006.02.002

Hald, G.M. (2006). Gender differences in pornography consumption among young heterosexual Danish adults. Archives of Sexual 
Behavior, 35(5), 577-585. doi:10.1007/s10508-006-9064-0

Henline, B., Lamke, L. \& Howard, M. (2007). Exploring perceptions of online infidelity. Personal Relationships, 14(1), 113-128. doi:10.1111/j.1475-6811.2006.00144.x

Henningsen, D. D. (2004). Flirting with Meaning: An examination of miscommunication in flirting interactions. Sex Roles, 50(7/8), 481-489. doi:10.1023/b:sers.0000023068.49352.4b

Henningsen, D.D., Braz, M. \& Davies, E. (2008). Why do we flirt?. Flirting motivations and sex differences in working and social contexts. Journal of Business Communication, 45(4), 483-502. doi:10.1177/0021943608319390

Hertlein, K. M. \& Piercy, F. P. (2012). Essential elements of Internet infidelity treatment. Journal of Marital and Family Therapy, 38(1), 257-270. doi:10.1111/j.1752-0606.2011.00275.x

Hertlein, K. M., \& Webster, M. (2008). Technology, relationships, and problems: A research synthesis. Journal of Marital and Family Therapy, 34(4) 445-460. doi:10.1111/j.1752-0606.2008.00087.x

Internet World Stats (2016). Internet Usage and Population Statistics for South America, Recuperado desde http://www.internetworldstats.com/stats $15 . h$ tm

Kholos, D. \& Childers, Ch. (2011). "Let my fingers do the talking”: Sexting and infidelity in -cyber space. Sexuality y Culture, 15(3) 217-239. doi:10.1007/s12119-011-9091-4

Klettke, B., Hallford, D. J., \& Mellor, D. J. (2014). Sexting prevalence and correlates: A systematic literature review. Clinical Psychology Review, 34(1), 44-53. doi:10.1016/j.cpr.2013.10.007

Leiblum, S., \& Döring, N. (2002). Internet sexuality: Known risks and fresh chances for women. En A. Cooper, (edit), Sex and the Internet: A guidebook for clinicians. (pp. 19-46). New York: Brunner-Routledge.

Maddox, A. M., Rhoades, G. K., \& Markman, H. J. (2011). Viewing sexually-explicit materials alone or together: Associations with relationship quality. Archives of Sexual Behavior, 40(2), 441-448. doi: 10.1007/s10508-009-9585-4

Manning, J. C. (2006). The Impact of Internet Pornography on Marriage and the Family: A review of the research. Sexual Addiction \& Compulsivity, 13(2-3), 131-165. doi:10.1080/10720160600870711

Millner, V. S. (2008). Internet Infidelity: A Case of Intimacy with Detachment. The Family Journal: Counseling and Therapy for Couples and Families, 16(1), 78-82. doi:10.1177/1066480707308918

Morgan, E. M. (2011). Associations between young adults' use of sexually explicit materials and their sexual preferences, behaviors, and satisfaction. Journal of Sex Research, 48(6), 520-530. doi: 10.1080/00224499.2010.543960

Nua Internet Surveys (October, 2003). How many online? [On-line] Extraído de: http://www.nua.ie/surveys/how_many_online/index.html Parker, T. S. \& Wampler, K. S. (2003). How bad is it? Perceptions of the relationship

impact of Internet sexual activities. Contemporary Family Therapy, 25(4), 415-429.

Resch, M. N. \& Alderson, K.G. (2014). Female partners of men who use pornography: are honesty and mutual use associated with relationship satisfaction?. Journal of Sex \& Marital Therapy. 40(5):410-424. doi: 10.1080/0092623X.2012.751077

Sabo, A.G. (2009). Highbrow and lowbrow pornography: Prejudice prevails against popular culture. A case study. The Journal of Popular Culture, 42(1), 147-161.

Scheinckman, M., \& Fishbane, M. D. (2004). The Vulnerability Cycle: Working with impasses in Couple Therapy. Family Process, 43(3) 279-299. doi:10.1111/j.1545-5300.2004.00023.x

Schneider, J. P. (2000). Effects of cybersex addiction on the family: Results of a survey. En: Cooper, A. (edt), Cybersex: The dark side of the force (pp. 31-58). Philadelphia: Brunner-Routledge.

Schneider, J. P. (2002). The New 'Elephant in the Living Room': Effects of Compulsive Cybersex Behaviors on the Spouse. En: Cooper, A. (edit) Sex and the Internet (pp. 169-186). New York: Brunner-Routledge.

Schneider, J. P. (2003). The impact of compulsive cybersex behaviours on the family. Sexual and Relationship Therapy, 18(3), 329-354. doi:10.1080/146819903100153946

Torrente, E., Piqueras, J.A., Orgilés, M., \& Espada, J.P. (2014). Asociación de la adicción a Internet con la ansiedad social y la falta de habilidades sociales en adolescentes españoles. Terapia Psicológica, 32(3), 175-184. doi:10.4067/s0718-48082014000300001

Træen, B., Spitznogle, K. \& Beverfjord, A. (2004). Attitudes and use of pornography in the Norwegian population 2002. Journal of Sex Research, 41(2), 193-200. doi:10.1080/00224490409552227

Vossler, A. (2016). Internet Infidelity 10 Years On: A Critical Review of the Literature. The Family Journal: Counseling and Therapy for Couples and Families, 24(4), 359-366. doi: 10.1177/1066480716663191

Wisman, M. A. (2016). Discovery of a Partner Affair and Major Depressive Episode in a Probability Sample of Married or Cohabiting Adults. Family Process, 55(4), 713-723. doi: 10.1111/famp.12185

Whitty, M. T. (2003). Cyber-flirting: Playing at love on the Internet. Theory and Psychology, 13(3) 339-357. doi:10.1177/0959354303013003003

Whitty, M. T. (2004). Cyber-flirting: An examination of men's and women's flirting behavior both offline and on the Internet. Behavior 
Change, 21(2) 115-126. doi.org/10.1375/bech.21.2.115.55423

Whitty, M. T. (2005). The realness of cybercheating: Men's and women's representations of unfaithful Internet relationships. Social Science Computer Review, 23(1), 57-67. doi;10.1177/0894439304271536

Whitty, M. T. (2007). Manipulation of self in cyberspace. En: Spitzberg, B. H. \& Cupach, W. R. (edts) The dark side of interpersonal communication (2nd ed.), (pp. 93-120). Mahwah, NJ: Erlbaum.

Whitty, M. T. \& Gavin, J. (2001). Age/sex/location: Uncovering the social cues in the development of online relationships. CyberPsychology and Behavior, 4(3) 623-630. doi:10.1089/109493101753235223

Whitty, M. T. \& Quigley, L. L. (2008). Emotional and sexual infidelity offline and in cyberspace. Journal of Marital and Family Therapy, 34(4), 461-468. doi: 10.1111/j.1752-0606.2008.00088.x

Young, K. \& Abreu, C. (2011). Internet addiction. A handbook and guide to evaluation and treatment. Hoboken, NJ: John Wiley \& Sons. Young, K. S., Griffin-Shelley, E., Cooper, A., O’Mara, J. \& Buchanan, J. (2000). Online infidelity: A new dimension in couple relationships with implications for evaluation and treatment. Sexual Addiction \& Compulsivity, 7(1-2), 59-74. doi:10.1080/10720160008400207 